\title{
Topical Treatment of Rosacea with Ivermectin Inhibits Gene Expression of Cathelicidin Innate Immune Mediators, LL-37 and KLK5, in Reconstructed and Ex Vivo Skin Models
}

\author{
Séverine Thibaut de Ménonville • Carine Rosignoli · Estelle Soares · \\ Manon Roquet • Béatrice Bertino · Jean-Paul Chappuis · Claire Defoin-Platel/Chaussade • \\ David Piwnica
}

Received: January 18, 2017 / Published online: February 27, 2017

(C) The Author(s) 2017. This article is published with open access at Springerlink.com

\section{ABSTRACT}

Introduction: Numerous intrinsic and extrinsic factors have been associated with the pathophysiology of rosacea, including dysregulation of innate immunity. A high level of cathelicidin antimicrobial peptides (e.g., LL-37) has been shown in the facial skin of patients with rosacea. Excessive production of both LL-37 and KLK5, the serine protease responsible for its cleavage, has been suggested to play a role in the pathophysiology of rosacea. Ivermectin $10 \mathrm{mg} / \mathrm{g}$ cream, indicated for the treatment of inflammatory lesions of rosacea, is reported to have dual anti-parasitic and anti-inflammatory properties. However, the exact mechanism of action of ivermectin cream in the treatment of rosacea is unknown.

Methods: This study aimed to evaluate the effect of ivermectin on the expression of KLK5 and the

Enhanced content To view enhanced content for this article go to http://www.medengine.com/Redeem/ 2097F06079268209.

Electronic supplementary material The online version of this article (doi:10.1007/s13555-017-0176-3) contains supplementary material, which is available to authorized users.

S. Thibaut de Ménonville $(\bowtie) \cdot$ C. Rosignoli .

E. Soares · M. Roquet · B. Bertino · J.-P. Chappuis

C. Defoin-Platel/Chaussade · D. Piwnica

Nestlé Skin Health R\&D, Sophia Antipolis, France e-mail:

Severine.THIBAUTDEMENONVILLE@galderma.com subsequent effect on the maturation process of cathelicidins. Experimental studies were performed either on normal human epidermal keratinocytes (NHEK), reconstructed human epidermis (RHE) or on human skin ex vivo stimulated with calcitriol $(1 \alpha, 25$-dihydroxyvitamin D3), which is known to induce KLK5 and LL-37 expression.

Results: The results show that ivermectin is able to inhibit KLK5 and CAMP gene expression and protein secretion in NHEK cells stimulated with calcitriol. Those results were confirmed in 3D models of the skin (RHE and skin ex vivo). The anti-inflammatory effects of ivermectin were associated with an inhibition of IL-8, IL-6 and MCP-1 (CCL2) secretion from NHEK cells. Conclusions: These results suggest that ivermectin can prevent the inflammatory effects of rosacea triggered by abnormal LL-37 processing, through the inhibition of $K L K 5$ gene expression in the epidermis.

Funding: Nestlé Skin Health R\&D.

Keywords: Cathelicidin; Innate immunity; Ivermectin; Rosacea

\section{INTRODUCTION}

Rosacea is an inflammatory disease of the facial skin, characterized by a variety of visible manifestations including erythema, papules, pustules and telangiectasias $[1,2]$. The 
pathophysiology of rosacea is yet to be fully elucidated; however, current evidence suggests that rosacea-prone skin inherently displays dysregulation of the neurovascular/neuroimmune system and the innate immune system [3-5]. The dysregulation of the innate immune system is supported by an augmentation of the cathelicidin innate immunity pathway [5-9].

Patients with rosacea express increased levels of cathelicidin (LL-37) in their facial skin compared with normal skin [10]. LL-37 is an antimicrobial peptide that provides innate defense against microbial organisms, and has been shown to promote cellular inflammation, as well as increased vascularity and angiogenesis $[11,12]$. The elevated levels of LL-37 observed in patients with rosacea are thought to result from unusually high levels of the serine protease kallikrein-5 (KLK5) $[6,10]$. KLK5 is responsible for the cleavage of the inactive precursor protein hCAP18 in the epidermis to form the active antimicrobial peptide LL-37 [6, 10]. LL-37 peptide fragments have been shown to control expression of extracellular matrix components, angiogenesis and leukocyte chemotaxis [11, 12]. Injection of these peptide fragments into the skin of mice led to an inflammatory phenotype similar to that seen in patients with rosacea [10]. Therefore, agents inhibiting the production of KLK5 or blocking its activity could prevent the expression of LL-37. This, in turn, could have inhibitory effects on the inflammatory cascade associated with rosacea [5].

Agents used in the treatment of rosacea, such as doxycycline, azelaic acid and metronidazole, have been shown to act on different targets within the inflammatory pathway [13]. The mechanism of action of two of these therapeutic agents has been linked to the inhibition of KLK5 [14, 15]. Oral doxycycline indirectly inhibits serine protease activity by inhibiting the matrix metalloproteinases involved in the activation of KLK5 [14]. Adults with papulopustular rosacea (PPR) were treated with doxycycline 40-mg modified-release capsules once daily for 12 weeks. LL-37 levels decreased over the course of 12 weeks of doxycycline treatment, and low levels of protease activity and LL-37 expression at 12 weeks correlated with clinical success [16]. Azelaic acid, a topical treatment for PPR, has been shown to directly inhibit KLK5 expression [15]. Nine-day treatment of mouse skin with azelaic acid $150 \mathrm{mg} / \mathrm{g}$ significantly decreased KLK5 messenger RNA (mRNA) expression compared with skin treated with vehicle control [15]. Adults with PPR who applied azelaic acid $150 \mathrm{mg} / \mathrm{g}$ twice daily showed significantly decreased CAMP (the gene encoding cathelicidin) mRNA after 4 weeks and decreased KLK5 mRNA after 12 weeks [15].

The exact mechanism of action of ivermectin cream $10 \mathrm{mg} / \mathrm{g}$, a new agent indicated for the treatment of inflammatory lesions of rosacea, remains unclear. However, it has been reported that ivermectin has both anti-inflammatory and anti-parasitic properties [17]. Ivermectin binds with high affinity to glutamate-gated chloride channels (commonly found in invertebrate nerve and muscle cells), resulting in hyperpolarization of the cells. This results in paralysis and death of parasites [17]. Importantly, ivermectin does not have this effect in human cells. Ivermectin also has anti-inflammatory effects; it has been shown to decrease humoral and cellular immune responses including neutrophil chemotaxis [18]. In order to further elucidate the mechanism of action of ivermectin and the inflammatory pathways that it targets, this study investigated the effect of ivermectin on the protein and gene expression of KLK5, LL-37 and pro-inflammatory markers including interleukin (IL)-8, IL-6 and monocyte chemoattractant protein (MCP)-1 [also known as chemokine (C-C motif) ligand (CCL) 2].

\section{METHODS}

\section{Normal Human Epidermal Keratinocytes}

Normal human epidermal keratinocytes (NHEKs) were isolated from abdominal plastic surgery at Nestlé Skin Health R\&D (Sophia Antipolis, France) as previously described [19-21] and cultured in Minimum Essential Media (MEM; Gibco, ThermoFisher Scientific, Waltham, MA, USA) medium supplemented with 10\% foetal bovine serum (FBS; Gibco), $10 \mathrm{ng} / \mathrm{ml}$ epidermal growth factor (Becton-Dickinson, Franklin Lakes, NJ, USA), 
$0.4 \mu \mathrm{g} / \mathrm{ml}$ of hydrocortisone, $1.8 \times 10^{-4} \mathrm{M}$ adenine, $5 \mu \mathrm{g} / \mathrm{ml}$ insulin, $5 \mu \mathrm{g} / \mathrm{ml}$ transferrin, $2 \mathrm{pM}$ tri-iodothyronin and $0.1 \mu \mathrm{g} / \mathrm{ml}$ cholera toxin (all from Sigma Aldrich, St. Louis, MO, USA), in an incubator at $37^{\circ} \mathrm{C}, 5 \% \mathrm{CO}_{2}$, and saturated humidity. At sub-confluence, cells were dissociated with tryspin-ethylenediaminetetraacetic acid (Gibco) and frozen in liquid nitrogen at $4 \times 10^{6}$ cells/vial in MEM growth medium supplemented with $10 \%$ dimethyl sulfoxide (DMSO) 20\% FBS. For the experiment $(n=6$ replicates/condition), NHEK cells were seeded at 10,000 cells $/ \mathrm{cm}^{2}$ in 48 -well culture plates in KGM-Gold medium (Lonza, Basel, Switzerland) with growth supplements. At confluence, media were removed from cells and replaced with media containing compounds at the indicated concentration during overnight incubation before stimulation either with calcitriol (1 $\alpha, 25$-dihydroxyvitamin D3; Sigma Aldrich) or LL-37 (Invivogen, San Diego, CA, USA). All treatments and stimulations were done in KGM-Gold medium (Lonza) with growth supplements. Cells were allowed to incubate for up to $48 \mathrm{~h}$. Media were collected after 24 and $48 \mathrm{~h}$ of stimulation and stored at $-20^{\circ} \mathrm{C}$ until analysis. Before gene expression analysis, cells were lysed using lysis buffer from Agencourt RNA advance Tissue Kit (Beckman Coulter, Bria, CA, USA) and stored at $-20{ }^{\circ} \mathrm{C}$ until ribonucleic acid (RNA) extraction.

\section{Reconstructed Human Epidermis}

Reconstructed human epidermis models (RHE; EpiSkin, L'Oréal, Lyon, France) $0.33 \mathrm{~cm}^{2} /$ HTS were purchased at D17 from Episkin (L'Oréal, Lyon, France). At reception, RHE D18 were removed from the agarose-nutrient solution in the multiwell plate and immediately placed in a plate in which each well had previously been filled with SkinEthic Growth Medium (EpiSkin, L'Oréal, Lyon, France) at room temperature. Culture dishes were placed in the incubator at $37^{\circ} \mathrm{C}, 5 \% \mathrm{CO}_{2}$ and saturated humidity. Testing could be initiated after at least $2 \mathrm{~h}$ resting. For the experiment ( $n=5$ replicates/condition), topical treatment with study compounds was applied to RHE D18 at $1 \mu \mathrm{l} / 0.1 \mathrm{~cm}^{2}$. After overnight incubation, a second treatment with the same compound was applied $1 \mathrm{~h}$ before RHE stimulation with topical calcitriol $(1 \alpha, 25$-dihydroxyvitamin D3; Sigma). RHE were allowed to incubate for up to $24 \mathrm{~h}$. Media was collected after $24 \mathrm{~h}$ and stored at $-20^{\circ} \mathrm{C}$ until analysis. Before gene expression analysis, RHE samples were lysed using lysis buffer from Agencourt RNA advance Tissue Kit (Beckman Coulter) and stored at $-20{ }^{\circ} \mathrm{C}$ until RNA extraction.

\section{Skin Ex Vivo}

Healthy human skin samples received at Nestlé Skin Health R\&D are derived from abdominoplasty surgery and obtained with the patient's consent and with local IRP approval. Skin biopsies were performed using 8-mm biopsy punches and placed on Transwell cell culture inserts, $24 \mathrm{~mm}$ diameter with a $8-\mu \mathrm{m}$ polycarbonate membrane (Costar; Sigma Aldrich) in 6-well plates containing KGM-Gold medium (Lonza) with growth supplements. For the experiment ( $n=3$ replicates/condition), surgical steel rings were deposited on the surface of the skin to allow topical treatment. Compounds were applied on skin ex vivo at $1 \mu \mathrm{l} / 0.1 \mathrm{~cm}^{2}$. After overnight incubation, a second treatment with the same compound was applied $1 \mathrm{~h}$ before skin stimulation either by topical treatment with calcitriol $(1 \alpha, 25$-dihydroxyvitamin D3; Sigma Aldrich) or with LL-37 (Invivogen). Biopsies were allowed to incubate for up to $72 \mathrm{~h}$. In order to proceed to histologic analysis on each replicate of treatment, a biopsy of $4 \mathrm{~mm}$ in diameter was performed inside the surgical steel rings. The sample was then tape-stripped ten times using D-squames discs (Monaderm, Monaco) and stored in 2-mL microtubes at $-80^{\circ} \mathrm{C}$ until protein analysis. Following tape-stripping, further 4-mm biopsies were carried out in order to perform gene expression and protein analysis.

\section{Gene Expression}

Cells were lysed using lysis buffer, and then stored at $-20^{\circ} \mathrm{C}$ until total RNA extraction was performed with the Agencourt RNA advance 
Tissue Kit (Beckman Coulter). RHE and skin biopsies were lysed in Tissue grinding CKMix50-R containing $600 \mu \mathrm{L}$ of lysis buffer and using Precellys-Dual (Ozyme, Montigny-le-Bretonneux, France). Reverse transcription was performed from $100 \mathrm{ng}$ of total RNA using the High Capacity Reverse transcription kit (High Capacity RNA-to-cDNA ${ }^{\mathrm{TM}}$ Kit; Applied Biosystems, Foster City, CA, USA). Pre-developed Taqman Assay probes were used to analyze gene expression on the Quantstudio $12 \mathrm{~K}$ Flex System (Applied Biosystems). Fold modulation relative to the control sample and adjusted $P$ value (False Discovery Rate Benjamini and Hochberg; FDR_BH) were calculated using the Array Studio software. "Undetermined" cycle threshold $(\mathrm{Ct})$ values were arbitrarily fixed at 40 to perform the analysis. Results were considered significant when at least a twofold difference in expression level was detected and statistical analysis revealed an adjusted $P$ value (FDR_BH) $<0.05$.

\section{Proteins Dosage}

Total protein was extracted from D-squames using $400 \mu \mathrm{L}$ Cell Lysis Buffer (Cell Signaling Technology, Danvers, MA, USA) in 2-mL Eppendorf microtubes. RHE and skin biopsies were lysed in Tissue grinding CKMix50-R containing $600 \mu \mathrm{L}$ of Cell Lysis Buffer (Cell Signaling Technology) and using Precellys-Dual (Ozyme, Montigny-le-Bretonneux, France). Total protein extract from RHE, skin biopsies and D-squames were quantified by a protein assay based on the Bradford method (Bio-Rad, Hercules, CA, USA). KLK5 protein (Human Kallikrein 5 Quantikine ELISA Kit; R\&D Systems, Minnneapolis, MN, USA), Cathepsin C (Antibodies-online, Atlanta, GA, USA) and LL-37 (Hycult biotech, Uden, The Netherlands) in NHEK culture media, RHE culture media and in skin ex vivo biopsies were measured by ELISA according to the manufacturer recommendation. Interleukin 8 (IL-8) secretion was quantified by HTRF (Human IL-8 Assay Kit; CisBio Assay, Codolet, France), according to the manufacturer's recommendations. ProcartaPlex Multiplex Luminex Immunoassays
(eBioscience, Affymetrix, Santa Clara, CA, USA) was used to enable simultaneous detection of 11 analytes in one sample. The analytes were glyceraldehyde 3-phosphate dehydrogenase $(\mathrm{GAPDH})$, chemokine (C-X-C motif) ligand (CXCL) 1, IL-6, CXCL10, CXCL11, chemokine (C-C motif) ligand (CCL) 2, CXCL9, CCL20, CCL5, soluble intercellular adhesion molecule (sICAM)-1, and tumur necrosis factor (TNF)- $\alpha$.

\section{Immunohistochemistry}

Tissues, either reconstructed epidermis or biopsies, were placed in fixative to preserve basic tissue architecture; they were then embedded in paraffin to support the tissue. Sections of $5 \mu \mathrm{m}$ were then cut and placed on a microscope slide for staining. Hematoxylin-eosin staining was performed on Symphony automate (Roche, Basel, Switzerland). Immunohistochemistry was performed on Discovery Xt, Ventana (Roche) using a rabbit anti-KLK5 antibody (TA321822; OriGene Technologies, Rockville, MD, USA) and a mouse anti-LL-37 antibody (sc-166770 clone D-5; Santa Cruz Biotechnology, Dallas, TX, USA).

\section{Compounds}

Calcitriol (1 $\alpha, 25$-dihydroxyvitamin D3) was purchased from Sigma Aldrich, and LL-37 from Invivogen and used following suppliers' recommendations. Ivermectin, azelaic acid and metronidazole were supplied by the chemical library management unit of Nestlé Skin Health R\&D. For systemic treatment, compounds were dissolved in DMSO at the desired concentrations, while $100 \%$ ethanol was used as vehicle for topical treatment.

\section{Statistical Analysis}

Statistical analysis was performed using bilateral and homoscedastic Student's $t$ test, and $P<0.05$ was considered to be statistically significant. The results are shown as mean \pm standard deviation (SD). 


\section{RESULTS}

\section{Effect of Ivermectin on Expression of KLK5 and the LL-37 Precursor Protein hCAP18}

To observe the effect of ivermectin on KLK5 secretion, the human epidermal cells were incubated overnight in media containing $1 \mu \mathrm{M}$ ivermectin, azelaic acid or metronidazole before being stimulated with calcitriol (Fig. 1a). In order to simulate the upregulation of KLK5 and LL-37 observed in the skin of patients with rosacea, NHEK cells were treated with $100 \mathrm{nM}$ calcitriol (the hormonally active metabolite of vitamin D) as previously described [22]. The
A

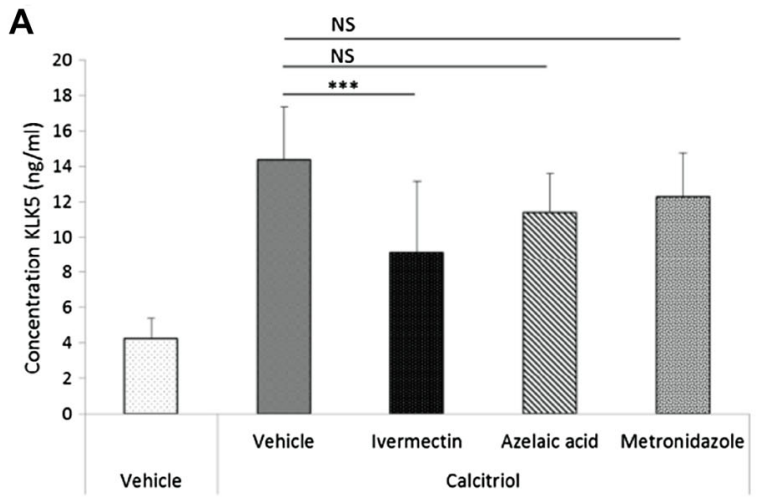

C

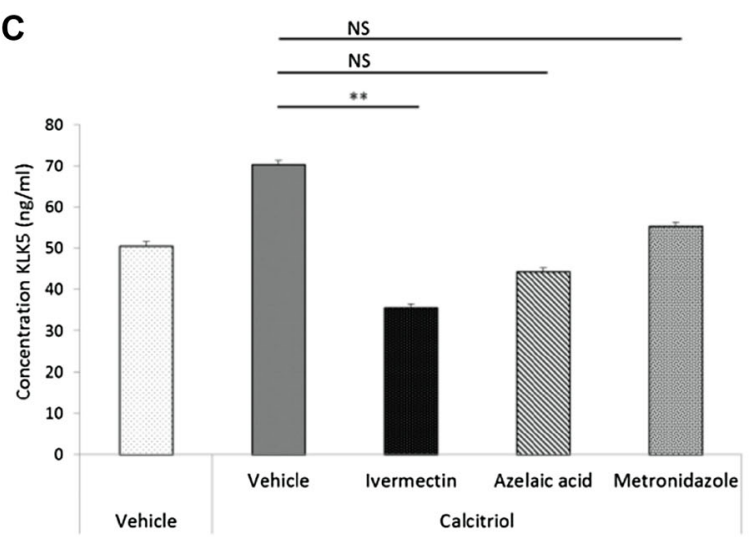

Fig. 1 Ivermectin treatment inhibits KLK5 secretion from human epidermal cells. a NHEK cells were pre-treated with ivermectin, azelaic acid, metronidazole at $1 \mu \mathrm{M}$ or vehicle control overnight before $48 \mathrm{~h}$ stimulation with $100 \mathrm{nM}$ calcitriol. KLK5 release was measured by ELISA. Data show mean \pm SD from 3 independent experiments. b RHE was pre-treated with ivermectin, azelaic acid, metronidazole at $1 \% \mathrm{w} / \mathrm{v}$ or vehicle control $(100 \%$ ethanol) overnight before $24 \mathrm{~h}$ topical stimulation with $10 \mu \mathrm{M}$ calcitriol. KLK5 release was measured by ELISA. Data show mean $\pm S D$ from two independent experiments. c Ex vivo skin biopsies were pre-treated with ivermectin, azelaic acid, metronidazole at $1 \% \mathrm{w} / \mathrm{v}$ or vehicle control overnight before $48 \mathrm{~h}$ topical stimulation
B

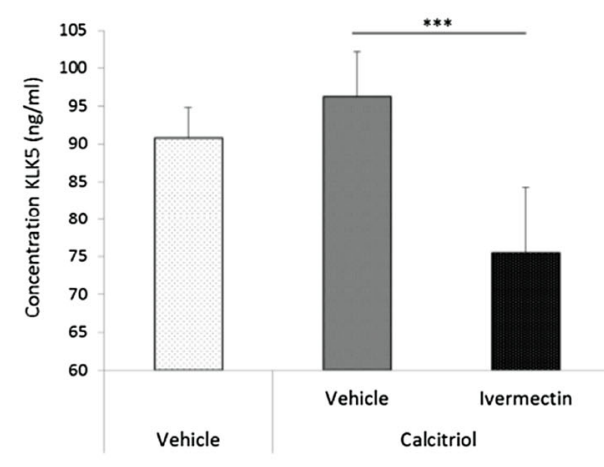

D

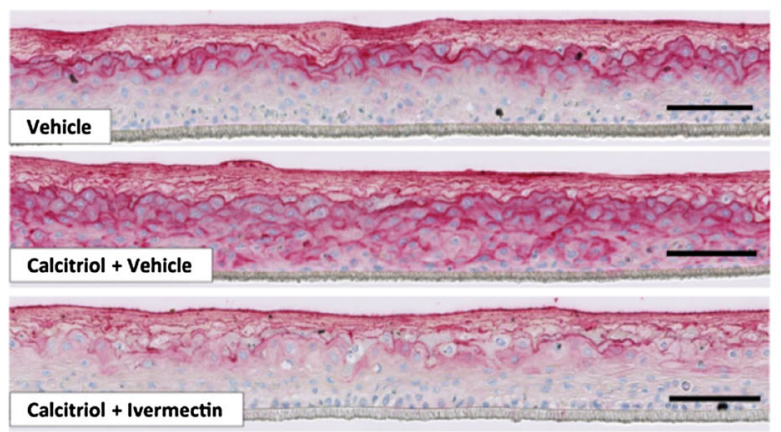

with $10 \mu \mathrm{M}$ calcitriol. KLK5 release was measured by ELISA. Data show mean $\pm S D$ from two independent experiments. $N S$ not significant. ${ }^{* *} P<.01,{ }^{* * *} P<.001$, Student's $t$ test. d Immunohistologic staining of paraffin-embedded reconstructed epidermis using anti-KLK5 antibody. Top panel RHE was pre-treated with vehicle control overnight and then was stimulated with vehicle control. Middle panel RHE was pre-treated with vehicle control overnight before being stimulated topically with $10 \mu \mathrm{M}$ calcitriol. Lower panel RHE was pre-treated with ivermectin at $1 \% \mathrm{w} / \mathrm{v}$ overnight before being stimulated topically with $10 \mu \mathrm{M}$ calcitriol. The epidermis was then paraffin-embedded and stained with anti-KLK5 antibody (in red). Scale bars $100 \mu \mathrm{m}$ 
supernatant was collected $48 \mathrm{~h}$ later and analyzed by ELISA for the presence of KLK5. No toxicity has been observed with compounds at the concentration tested (data not shown). Pre-treatment with ivermectin resulted in a significant decrease in the concentration of KLK5 secreted from NHEK cells $(P<0.001$; Fig. 1a) compared with vehicle control (DMSO). However, no significant difference was observed when NHEK cells were treated with azelaic acid or metronidazole (Fig. 1a).

This experiment was then repeated using topical ivermectin on RHE, which is the clinically relevant application method. Ivermectin $1 \% \mathrm{w} / \mathrm{v}$ was applied to a RHE layer and left to incubate overnight. A second treatment was given $1 \mathrm{~h}$ before RHE stimulation with $10 \mu \mathrm{M}$ calcitriol. The supernatant was collected $24 \mathrm{~h}$ later and analyzed by ELISA for the presence of KLK5. Treatment with topical ivermectin resulted in a significant decrease in the concentration of KLK5 secreted from RHE cells $(P<0.001)$ compared with vehicle control (ethanol; Fig. 1b). Similarly, two treatments with topical ivermectin significantly decreased the amount of KLK5 secreted from ex vivo human skin biopsies after stimulation with calcitriol $(P=0.006)$ compared with vehicle control (Fig. 1c, Supplementary Fig. 1). Histologic analysis (data not shown) has been performed in order to ensure that ivermectin did not affect the integrity of ex vivo human skin biopsies. No significant difference in KLK5 secretion was observed after topical treatment with azelaic acid or metronidazole (Fig. 1c).

To confirm these results, immunohistologic staining of paraffin-embedded reconstructed epidermis was carried out. Analysis of KLK5 expression by the differentiated keratinocytes showed an increase in KLK5 after stimulation with calcitriol, which was substantially inhibited by pre-treatment with topical ivermectin (Fig. 1d, qualitative assessment). This indicates that ivermectin significantly inhibits the intracellular expression and secretion of KLK5 from keratinocytes, both in vitro and ex vivo. As previously mentioned, KLK5 is responsible for the cleavage of the inactive precursor protein hCAP18 in the epidermis to form the active antimicrobial peptide LL-37 $[6,10]$.
In order to assess whether ivermectin has an effect on the expression of hCAP18 in keratinocytes, the cells were incubated overnight in media containing $1 \mu \mathrm{M}$ ivermectin, azelaic acid or metronidazole before being stimulated with calcitriol. The supernatant was collected $48 \mathrm{~h}$ later and analyzed by ELISA for the presence of hCAP18. Pre-treatment with ivermectin resulted in a decrease in the concentration of hCAP18 secreted from NHEK cells compared with vehicle control (DMSO), but this difference was not statistically significant (Fig. 2a). This decrease can be observed more dramatically by immunohistologic analysis of hCAP18/LL-37 expression in RHE cells (Fig. 2b, qualitative assessment). Calcitriol stimulation substantially increases the level of hCAP18 staining (indicated by black arrows) observed in the differentiated keratinocytes compared to vehicle control. Pre-treatment with ivermectin resulted in an inhibition of hCAP18/LL-37 protein expression, resulting in staining that is comparable to vehicle control (Fig. 3b). Taken together, these results indicate that pre-treatment with ivermectin inhibits the expression of KLK5 and the LL-37 precursor protein hCAP18.

\section{Effect of Ivermectin on Cytokine Secretion}

Ivermectin has been previously shown to inhibit multiple pro-inflammatory cytokines which are downstream of LL-37 activation, including IL-6 and TNF- $\alpha$ [23]. However, the effect of ivermectin on IL-8, the pro-inflammatory cytokine responsible for neutrophil recruitment $[24,25]$, has yet to be elucidated. Here, we pre-treated NHEK cells with ivermectin, azelaic acid, metronidazole or vehicle control before stimulating them with calcitriol, and then measured IL-8 secretion after $48 \mathrm{~h}$. Ivermectin significantly reduced IL- 8 response at $48 \mathrm{~h}$ compared with vehicle control $(P<0.001$; Fig. 3a). This inhibition of IL-8 secretion was not observed after pre-treatment with azelaic acid or metronidazole. These results were confirmed in 3D models of the skin, where RHE and ex vivo skin biopsies were pre-treated with topical ivermectin or vehicle control and then stimulated with calcitriol (Fig. 3b, c). 


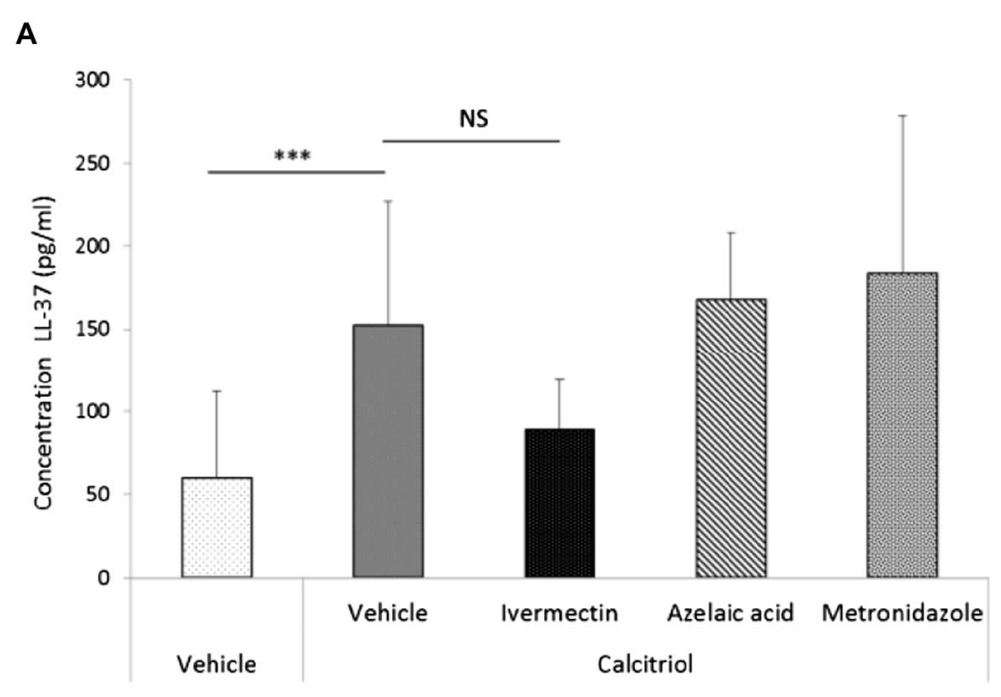

B

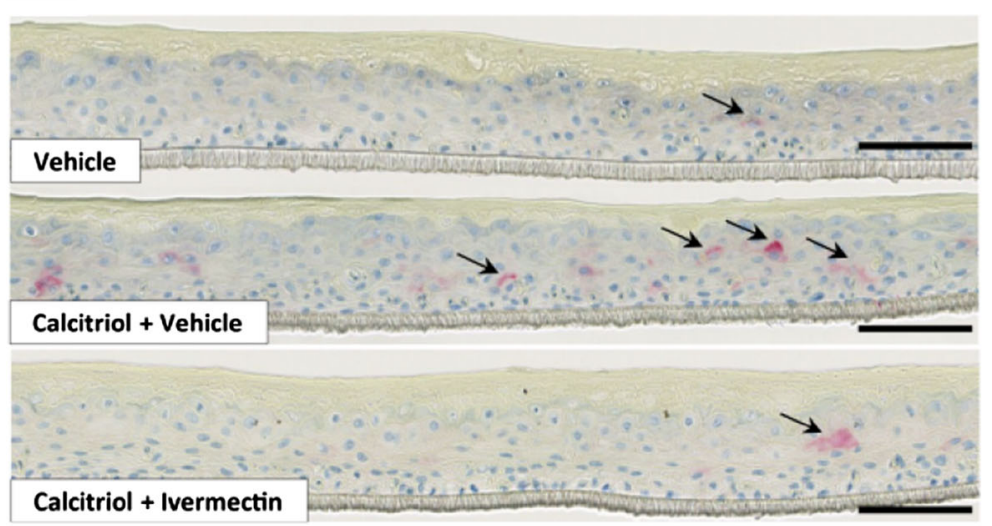

Fig. 2 Ivermectin treatment inhibits LL-37 expression in human epidermal cells. a NHEK cells were pre-treated with vehicle control (100\% ethanol) or $1 \mu \mathrm{M}$ ivermectin, azelaic acid or metronidazole overnight before $48 \mathrm{~h}$ stimulation with $100 \mathrm{nM}$ calcitriol. hCAP18/LL-37 release was measured by ELISA. Data show mean \pm SD from two independent replicates. NS not significant. ${ }^{* * *} P<.001$, Student's $t$ test. $\mathbf{b}$ Immunohistologic staining of paraffin-embedded reconstructed epidermis using

Stimulation of keratinocytes with LL-37 has been shown to induce keratinocyte migration, proliferation and production of pro-inflammatory cytokines [26]. Therefore, we evaluated the effect of ivermectin on secretion of IL-8 after stimulation with LL-37. Ivermectin significantly decreased the secretion of IL-8 from NHEK cells
anti-LL-37 antibody (in red). Top panel RHE was pre-treated with vehicle control overnight and then was stimulated with vehicle control. Middle panel RHE was pre-treated with vehicle control overnight before being stimulated topically with $10 \mu \mathrm{M}$ calcitriol. Lower panel RHE was pre-treated with ivermectin overnight before being stimulated with calcitriol. The epidermis was then paraffin-embedded and stained with anti-LL-37 antibody (in red). Scale bars $100 \mu \mathrm{m}$

compared with vehicle control after stimulation with LL-37 (Fig. 3d). However, pre-treatment with azelaic acid or metronidazole had no effect on IL-8 secretion. Overall, these results suggest that pre-treatment with ivermectin inhibits the secretion of IL-8 after stimulation with both calcitriol and LL-37. 

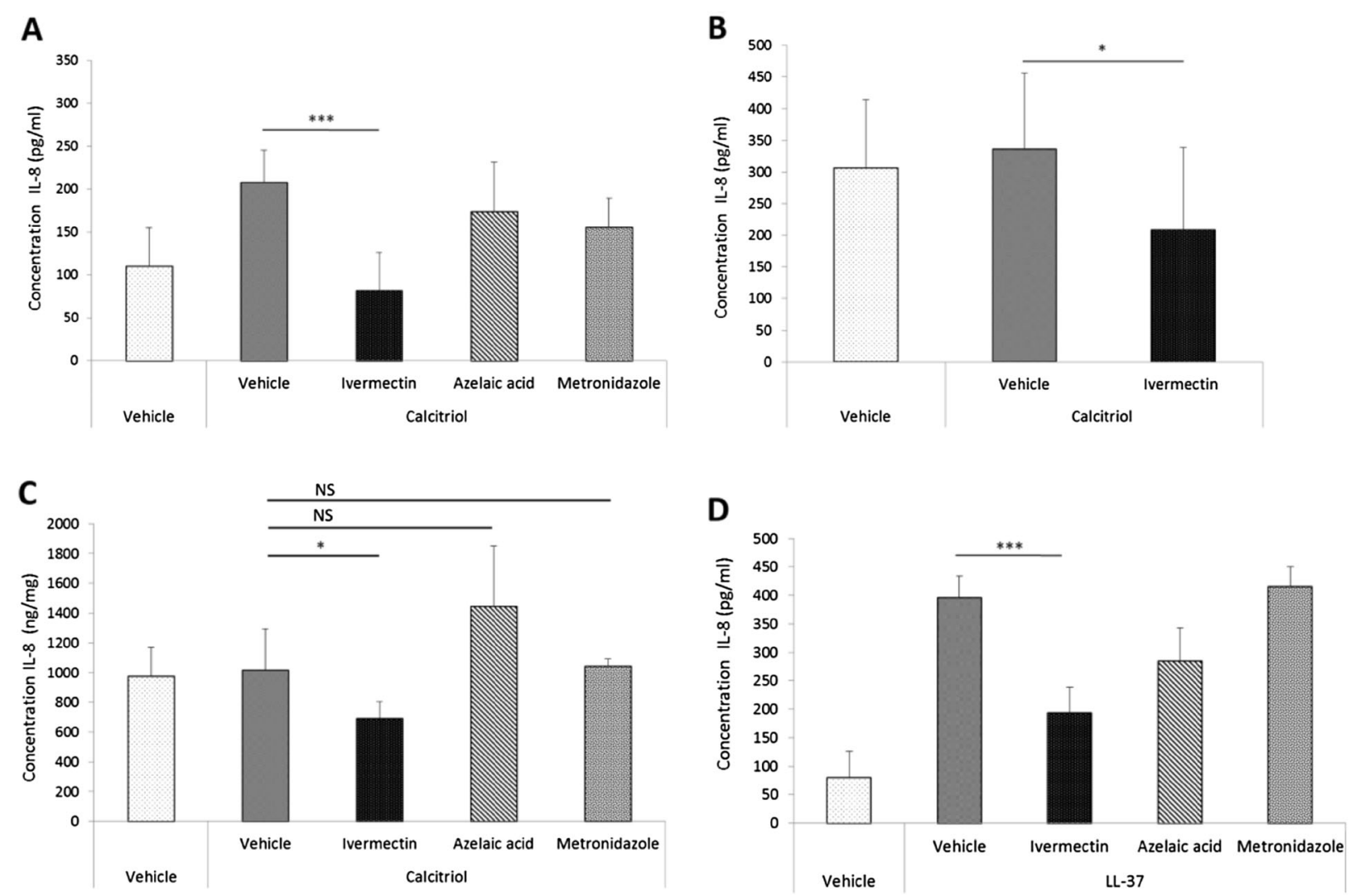

Fig. 3 Ivermectin treatment inhibits IL-8 secretion from human epidermal cells. a NHEK cells were pre-treated with vehicle control (100\% ethanol) or $1 \mu \mathrm{M}$ ivermectin, azelaic acid or metronidazole overnight before $48 \mathrm{~h}$ stimulation with $100 \mathrm{nM}$ calcitriol. IL-8 release was measured by HTRF. Data show mean \pm SD from 6 independent replicates. b RHE was pre-treated with ivermectin, azelaic acid, metronidazole at $1 \% \mathrm{w} / \mathrm{v}$ or vehicle control overnight before $24 \mathrm{~h}$ topical stimulation with $10 \mu \mathrm{M}$ calcitriol. IL-8 release was measured by HTRF. Data show mean $\pm S D$ from two independent

To assess the effect of ivermectin on other cytokines present in the skin, multiplex immunoassays were carried out on ex vivo skin biopsies. Only the levels of IL-6, CCL2, CXCL10 and soluble ICAM-1 were sufficient to be quantifiable in the assays. The results show that ivermectin significantly inhibits the secretion of IL-6 and CCL2 (Supplementary Fig. 2). Taken together, these results indicate that ivermectin significantly inhibits the signaling pathway downstream of cathelicidin.

experiments. c Ex vivo skin biopsies were pre-treated with ivermectin, azelaic acid, metronidazole at $1 \% \mathrm{w} / \mathrm{v}$ or vehicle control overnight before $48 \mathrm{~h}$ topical stimulation with $10 \mu \mathrm{M}$ calcitriol. IL-8 release was measured by HTRF. Data show mean $\pm S D$ from two independent experiments. d NHEK cells were pre-treated with ivermectin, azelaic acid, metronidazole or vehicle control overnight before $24 \mathrm{~h}$ stimulation with LL-37 $25 \mu \mathrm{g} / \mathrm{ml}$. IL-8 release was measured by HTRF. Data show mean \pm SD from two independent experiments. NS not significant. ${ }^{* *} P<.01,{ }^{* * *} P<.001$, Student's $t$ test

\section{Effect of Ivermectin on Gene Expression of KLK5 and Cathelicidin}

It has been previously shown that treatment with azelaic acid directly affects the gene expression of KLK5 and cathelicidin [15]. It is therefore possible that the inhibition of KLK5, LL-37 and IL-8 protein expression reported in this study could be due to a direct effect on KLK5 and CAMP gene expression. In order to verify this hypothesis, we evaluated whether 
ivermectin inhibits KLK5, LL-37 and IL-8 mRNA expression after stimulation with calcitriol. Human ex vivo skin biopsies were pre-treated with topical ivermectin or ethanol as vehicle control and then stimulated with calcitriol for either 24 or $72 \mathrm{~h}$. Topical treatment with ivermectin significantly and selectively inhibits CAMP gene expression induced by 24 -h stimulation with calcitriol. No other genes encoding markers of inflammation were affected by the treatment with ivermectin. The effect on $K L K 5$ gene expression was less pronounced, but showed a significant decrease after 72-h stimulation with calcitriol (Fig. 4). This effect was specific to $K L K 5$ gene expression and did not affect the genetic expression of other skin-expressed proteases. This experiment was also carried out in the RHE system, although only 24-h stimulation with calcitriol was tested, showing similar inhibition of CAMP gene expression with topical ivermectin treatment (Supplementary Table 1). These results provide clear evidence that ivermectin inhibits KLK5 and LL-37 expression at the gene level, with no effect on IL-8 gene expression. This effect of ivermectin was associated with inhibition of IL-8, IL-6 and CCL2 secretion, suggesting that ivermectin prevents activation of the inflammatory pathways involved in the pathophysiology of rosacea.

\section{DISCUSSION}

Augmentation of the cathelicidin innate immune pathway (specifically KLK5 and LL-37) has been shown to be a major contributor to the pathophysiology of rosacea $[6,10]$. Agents preventing the production of KLK5 and hCAP18, and thereby inhibiting the activation of LL-37, could have a considerable impact on the inflammation affiliated with rosacea. In the present study, we demonstrate for the first time that ivermectin inhibits the expression of KLK5 and the inactive precursor protein of LL-37, hCAP18, at the gene level in calcitriol-induced keratinocytes, both in vitro and ex vivo. These results suggest that ivermectin may act at the gene level to prevent the activation of inflammatory pathways involved in the pathophysiology of rosacea. Interestingly, however, previous studies performed with doxycycline and ivermectin (data not published) have demonstrated that ivermectin did not affect the gene expression of matrix metalloproteinases involved in the proteolytic activation of KLK5.

Earlier studies have shown that facial skin from patients with rosacea overexpress KLK5 and cathelicidin compared with normal skin [10]. LL-37 has been shown to be pro-inflammatory in several experimental systems [11, 12], and the current hypothesis is that the augmentation of the cathelicidin immune pathway is a major contributor to symptoms observed in rosacea [16]. In light of this, inhibition of cathelicidin has been suggested to correlate directly with beneficial therapeutic activity in patients with rosacea. Indeed, azelaic acid, a commonly used topical therapy for rosacea, has been shown to directly inhibit KLK5 and cathelicidin gene expression [15]. In this study, the decrease in KLK5 and LL-37 protein expression observed after pre-treatment with azelaic acid did not reach statistical significance. Importantly, however, the levels of KLK5 and LL-37 expression after ivermectin treatment were lower than that observed with azelaic acid, showing increased inhibition (Figs. 1, 3). This indicates that ivermectin has a greater effect on the cathelicidin immune pathway than has been observed with other effective topical treatments for rosacea.

This inhibition of cathelicidin expression from keratinocytes correlates well with the previously reported long-term safety and efficacy of ivermectin treatment. In two 40-week extension studies assessing the safety and tolerability profile of ivermectin versus azelaic acid in PPR patients over the long term, the ivermectin cream $10 \mathrm{mg} / \mathrm{g}$ treatment group had a lower incidence of treatment-related adverse events compared with the azelaic acid $150 \mathrm{mg} / \mathrm{g}$ treatment group [27]. In addition, ivermectin has a better long-term tolerability profile compared with azelaic acid. The majority of patients treated with ivermectin reported no stinging, dryness or itching, whereas a majority of patients treated with azelaic acid reported some of these symptoms [27]. 


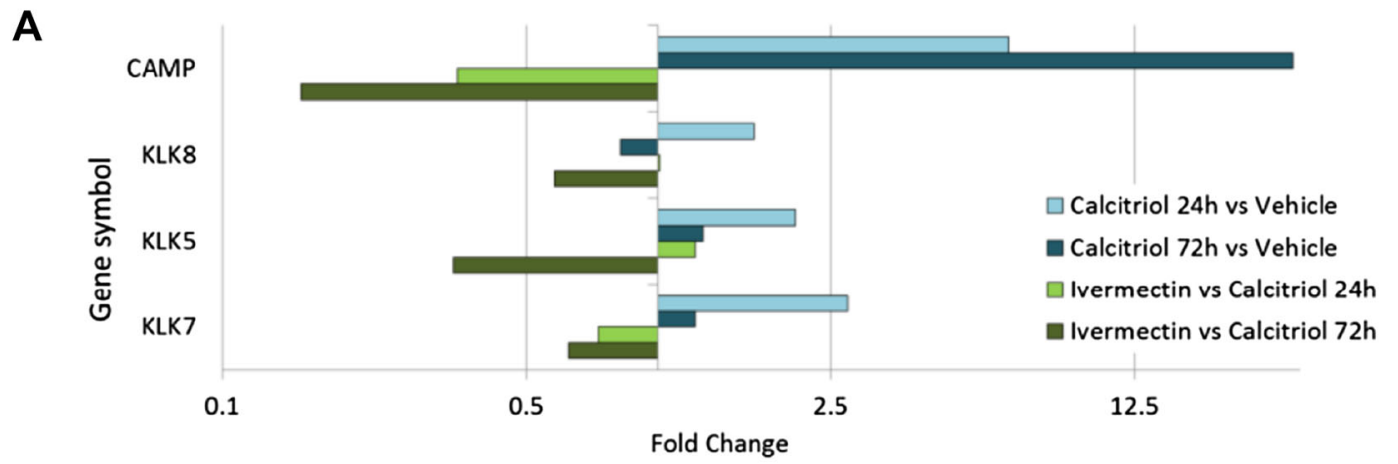

B

\begin{tabular}{|c|c|c|c|c|c|c|c|c|c|c|}
\hline \multirow{3}{*}{ Gene symbol } & \multirow{3}{*}{$\begin{array}{l}\text { Normalized Ct } \\
\text { Vehicle 24h }\end{array}$} & \multicolumn{4}{|c|}{$24 \mathrm{~h}$} & \multirow{3}{*}{$\begin{array}{l}\text { Normalized Ct } \\
\text { Vehicle } 72 \mathrm{~h}\end{array}$} & \multicolumn{4}{|c|}{$72 \mathrm{~h}$} \\
\hline & & \multicolumn{2}{|c|}{ Calcitriol vs Vehicle } & \multicolumn{2}{|c|}{$\begin{array}{c}\text { Calcitriol + } \\
\text { Ivermectin vs Vehicle }\end{array}$} & & \multicolumn{2}{|c|}{ Calcitriol vs Vehicle } & \multicolumn{2}{|c|}{$\begin{array}{c}\text { Calcitriol + } \\
\text { Ivermectin vs Vehicle }\end{array}$} \\
\hline & & Fold Change & FDR_BH & Fold Change & FDR_BH & & Fold Change & FDR_BH & Fold Change & FDR_BH \\
\hline \multicolumn{11}{|l|}{ Skin protease } \\
\hline KLK7 & 26.9 & 2.74 & 4.83E-07 & 0.73 & 0.6308 & 24.3 & 1.22 & 0.7533 & 0.63 & 0.5147 \\
\hline KLK5 & 25.9 & 2.07 & 0.0002 & 1.22 & 0.8042 & 22.4 & 1.27 & 0.7533 & 0.34 & 0.0155 \\
\hline KLK8 & 26.8 & 1.67 & ( 0.0188 & 1.01 & 0.9784 & 24.4 & 0.82 & 0.7533 & 0.58 & 0.453 \\
\hline SERPINA5 & 35.6 & 1.66 & 0.388 & 1.17 & 0.9535 & 35.1 & 0.68 & 0.7533 & 0.60 & 0.8207 \\
\hline MMP2 & 30.6 & 1.32 & 0.9445 & 0.74 & 0.9535 & 39.3 & 0.72 & 0.962 & 1.33 & 0.9531 \\
\hline ELANE & 38.6 & 1.25 & 0.7757 & 0.90 & 0.9535 & 37.5 & 0.44 & 0.6622 & 0.86 & 0.9531 \\
\hline MMP9 & 33.1 & 1.12 & 0.8279 & 1.18 & 0.8042 & 34.1 & 1.84 & 0.5496 & 0.52 & 0.453 \\
\hline TPSB2;TPSAB1 & 30.8 & 1.09 & 0.867 & 1.04 & 0.9535 & 30.1 & 0.42 & 0.2866 & 0.80 & 0.9051 \\
\hline SPINK6 & 40.4 & 1.08 & 0.7653 & 0.99 & 0.9784 & 39.3 & 1.15 & 0.7533 & 1.10 & 0.9051 \\
\hline KLK14 & 32.2 & 1.04 & 0.9489 & 0.79 & 0.8042 & 31.1 & 0.83 & 0.8891 & 0.81 & 0.9531 \\
\hline CMA1 & 34.2 & 1.01 & 0.9979 & 1.42 & 0.8042 & 33.9 & 0.64 & 0.7533 & 0.39 & 0.453 \\
\hline SPINKS & 24.9 & 0.90 & 0.7757 & 1.41 & 0.2827 & 22.4 & 0.84 & 0.7533 & 0.49 & 0.1352 \\
\hline SERPINF2 & 34.8 & 0.89 & 0.7467 & 1.09 & 0.9056 & 34.2 & 0.64 & 0.4212 & 0.96 & 0.9531 \\
\hline MMP20 & 37.1 & 0.72 & 0.9445 & 1.46 & 0.9535 & 28.3 & 1.15 & 0.962 & 1.10 & 0.9739 \\
\hline \multicolumn{11}{|c|}{ Inflammation markers } \\
\hline CAMP & 39.7 & 6.42 & W1.89E-07 & 0.35 & 0.0102 & 39.1 & 28.93 & - $9.13 \mathrm{E}-07$ & 0.15 & 0.0155 \\
\hline TNF & 31.5 & 1.21 & 0.7653 & 1.35 & 0.8042 & 31.7 & 0.90 & 0.8891 & 1.12 & 0.9531 \\
\hline TSLP & 31.0 & 1.11 & 0.7872 & 0.81 & 0.8042 & 28.9 & 0.98 & 0.962 & 0.63 & 0.5147 \\
\hline TLR2 & 30.6 & 1.04 & 0.9445 & 1.20 & 0.8042 & 28.3 & 0.92 & 0.8891 & 0.72 & 0.8207 \\
\hline ICAM1 & 30.8 & 0.96 & 0.9445 & 0.94 & 0.9535 & 31.2 & 0.81 & 0.7533 & 1.21 & 0.9051 \\
\hline F2RL1 & 28.9 & 0.80 & 0.678 & 0.92 & 0.9535 & 28.3 & 0.46 & 0.3857 & 1.06 & 0.9531 \\
\hline 118 & 28.7 & 0.63 & 0.678 & 0.94 & 0.9535 & 30.1 & 0.42 & 0.7533 & 1.92 & 0.8207 \\
\hline TLR9 & 30.8 & 0.62 & 0.678 & 1.37 & 0.8042 & 29.9 & 1.35 & 0.8891 & 0.64 & 0.9051 \\
\hline FPR2 & 37.5 & 0.49 & 0.678 & 0.75 & 0.9535 & 37.8 & 1.42 & 0.8891 & 0.69 & 0.9531 \\
\hline TLR7 & 37.1 & 0.44 & 0.678 & 0.83 & 0.9535 & 37.2 & 0.46 & 0.7533 & 1.28 & 0.9531 \\
\hline \multicolumn{11}{|c|}{ Differentiation Markers } \\
\hline IVL & 28.2 & 1.07 & 0.9445 & 0.74 & 0.8042 & 25.2 & 0.59 & 0.7533 & 0.61 & 0.8207 \\
\hline TGM1 & 27.7 & 1.00 & 0.9979 & 1.23 & 0.8042 & 23.5 & 0.88 & 0.8891 & 0.45 & 0.453 \\
\hline FLG & 24.9 & 0.49 & 0.1139 & 1.00 & 0.9874 & 21.9 & 0.33 & 0.4212 & 0.59 & 0.8207 \\
\hline \multicolumn{11}{|c|}{ House Keeping Gene } \\
\hline HPRT1 & 28.9 & 1.17 & 0.1908 & 0.95 & 0.9056 & 29.4 & 0.88 & 0.7533 & 1.12 & 0.8536 \\
\hline GUSB & 28.6 & 0.95 & 0.867 & 1.14 & 0.8042 & 28.1 & 0.86 & 0.7533 & 0.89 & 0.9051 \\
\hline GAPDH & 23.0 & 0.84 & 0.6985 & 0.85 & 0.8042 & 23.1 & 1.67 & 0.5496 & 1.03 & 0.9531 \\
\hline
\end{tabular}

Fig. 4 Ivermectin treatment inhibits cathelicidin pathway gene expression in ex vivo human skin biopsies. Ex vivo skin biopsies were pre-treated with ivermectin at $1 \% \mathrm{w} / \mathrm{v}$ or vehicle control (100\% ethanol) before either 24- or 72 -h topical stimulation with $10 \mu \mathrm{M}$ calcitriol. Tissues were lysed and RNA extracted in order to perform qRT-PCR using pre-developed Taqman Assay probes. a Graphical representation show fold change modulation of CAMP, KLK8, KLK7 and KLK5 gene expression after 24-h stimulation and $72-\mathrm{h}$ stimulation. b Table represents normalized $\mathrm{Ct}$ values considering housekeeping gene expression (GAPDH, HPRT1 and GUSB) of control sample (vehicle-treated) at 24 or $72 \mathrm{~h}$. The lower is the normalized $\mathrm{Ct}$ value; the higher is the gene expression value. Fold induction are calculated for calcitriol-treated sample using vehicle as reference sample. Data show mean from three independent experiments. Analyses were performed using Array Studio software. Results were considered significant when at least a twofold difference in expression level was detected and statistical analysis revealed an adjusted $P$ value $($ FDR_BH) $<0.05$ 
The long-term efficacy associated with ivermectin was recently demonstrated in an extension of the Phase III superiority study of ivermectin versus metronidazole. The results of this study showed that initial successful treatment with ivermectin cream $10 \mathrm{mg} / \mathrm{g}$ significantly extended the remission of rosacea compared with metronidazole cream when treatment was stopped [28]. The effects of ivermectin on KLK5 and CAMP gene expression ex vivo could explain the increased remission time seen with ivermectin treatment versus metronidazole, and this could indicate an impact of this agent on the course of the disease. It is important to recognise, however, that these experiments were conducted in vitro and ex vivo. Further studies are needed to confirm these findings in rosacea patients with papulopustular lesions.

\section{CONCLUSIONS}

Overall, the results of this study suggest that ivermectin treatment prevents the augmentation of the cathelicidin immune pathway, which has a positive impact on the downstream inflammatory pathway (e.g., decreased IL-8, IL- 6 and CCL2 secretion). These results suggest that ivermectin can prevent the inflammatory effects of rosacea triggered by abnormal LL-37 processing, through the inhibition of KLK5 and $C A M P$ gene expression in the epidermis.

\section{ACKNOWLEDGEMENTS}

Funding for the study and article processing charges was provided by Nestlé Skin Health R\&D, Sophia Antipolis, France. All authors had full access to all of the data in this study and take complete responsibility for the integrity of the data and accuracy of the data analysis. All named authors meet the International Committee of Medical Journal Editors (ICMJE) criteria for authorship for this manuscript, take responsibility for the integrity of the work as a whole, and have given final approval for the version to be published. Medical writing support was provided by Dr. Kathryn Lagrue (Medical Writer) and Delia
Freeman (Associate Director) of Havas Life Medicom, and was funded by Nestlé Skin Health R\&D, Sophia Antipolis, France.

Disclosures. Séverine Thibaut de Ménonville, Carine Rosignoli, Estelle Soares, Manon Roquet, Béatrice Bertino, Jean-Paul Chappuis, Claire Defoin-Platel/Chaussade and David Piwnica have nothing to disclose.

Compliance with ethics guidelines. Healthy human skin samples received at Nestlé Skin Health R\&D (Sophia Antipolis, France) are derived from abdominoplasty surgery and obtained with the patient's consent and with local IRP approval.

Data availability. The datasets analysed during the current study are available from the corresponding author on reasonable request.

Open Access. This article is distributed under the terms of the Creative Commons Attribution-NonCommercial 4.0 International License (http://creativecommons.org/licenses/ by-nc/4.0/), which permits any noncommercial use, distribution, and reproduction in any medium, provided you give appropriate credit to the original author(s) and the source, provide a link to the Creative Commons license, and indicate if changes were made.

\section{REFERENCES}

1. Crawford GH, Pelle MT, James WD. Rosacea: I. Etiology, pathogenesis, and subtype classification. J Am Acad Dermatol. 2004;51(3):327-41 (quiz 42-44).

2. Steinhoff M, Buddenkotte J, Aubert J, Sulk M, Novak P, Schwab VD, et al. Clinical, cellular, and molecular aspects in the pathophysiology of rosacea. J Investig Dermatol Symp Proc. 2011;15(1):2-11.

3. Schwab VD, Sulk M, Seeliger S, Nowak P, Aubert J, Mess C, et al. Neurovascular and neuroimmune aspects in the pathophysiology of rosacea. J Investig Dermatol Symp Proc. 2011;15(1):53-62.

4. Del Rosso JQ, Gallo RL, Kircik L, Thiboutot D, Baldwin HE, Cohen D. Why is rosacea considered to be an inflammatory disorder? The primary role, 
clinical relevance, and therapeutic correlations of abnormal innate immune response in rosacea-prone skin. J Drugs Dermatol. 2012;11(6):694-700.

5. Two AM, Del Rosso JQ. Kallikrein 5-mediated inflammation in rosacea: clinically relevant correlations with acute and chronic manifestations in rosacea and how individual treatments may provide therapeutic benefit. J Clin Aesthet Dermatol. 2014;7(1):20-5.

6. Yamasaki K, Schauber J, Coda A, Lin H, Dorschner RA, Schechter NM, et al. Kallikrein-mediated proteolysis regulates the antimicrobial effects of cathelicidins in skin. FASEB J. 2006;20(12):2068-80.

7. Meyer-Hoffert U, Schroder JM. Epidermal proteases in the pathogenesis of rosacea. J Investig Dermatol Symp Proc. 2011;15(1):16-23.

8. Yamasaki K, Gallo RL. Rosacea as a disease of cathelicidins and skin innate immunity. J Investig Dermatol Symp Proc. 2011;15(1):12-5.

9. Yamasaki K, Kanada K, Macleod DT, Borkowski AW, Morizane S, Nakatsuji T, et al. TLR2 expression is increased in rosacea and stimulates enhanced serine protease production by keratinocytes. J Invest Dermatol. 2011;131(3):688-97.

10. Yamasaki K, Di Nardo A, Bardan A, Murakami M, Ohtake T, Coda A, et al. Increased serine protease activity and cathelicidin promotes skin inflammation in rosacea. Nat Med. 2007;13(8):975-80.

11. De Y, Chen Q, Schmidt AP, Anderson GM, Wang JM, Wooters J, et al. LL-37, the neutrophil granuleand epithelial cell-derived cathelicidin, utilizes formyl peptide receptor-like 1 (FPRL1) as a receptor to chemoattract human peripheral blood neutrophils, monocytes, and $\mathrm{T}$ cells. J Exp Med. 2000;192(7):1069-74.

12. Koczulla R, von Degenfeld G, Kupatt C, Krotz F, Zahler S, Gloe T, et al. An angiogenic role for the human peptide antibiotic LL-37/hCAP-18. J Clin Invest. 2003;111(11):1665-72.

13. Salzer S, Ruzicka T, Schauber J. Face-to-face with anti-inflammatory therapy for rosacea. Exp Dermatol. 2014;23(6):379-81.

14. Kanada KN, Nakatsuji T, Gallo RL. Doxycycline indirectly inhibits proteolytic activation of tryptic kallikrein-related peptidases and activation of cathelicidin. J Invest Dermatol. 2012;132(5):1435-42.

15. Coda AB, Hata T, Miller J, Audish D, Kotol P, Two A, et al. Cathelicidin, kallikrein 5 , and serine protease activity is inhibited during treatment of rosacea with azelaic acid 15\% gel. J Am Acad Dermatol. 2013;69(4):570-7.
16. Di Nardo A, Holmes AD, Muto Y, Huang EY, Preston $\mathrm{N}$, Winkelman WJ, et al. Improved clinical outcome and biomarkers in adults with papulopustular rosacea treated with doxycycline modified-release capsules in a randomized trial. J Am Acad Dermatol. 2016;74(6):1086-92.

17. Abokwidir M, Fleischer AB. An emerging treatment: topical ivermectin for papulopustular rosacea. J Dermatolog Treat. 2015;26(4):379-80.

18. Yan S, Ci X, Chen N, Chen C, Li X, Chu X, et al. Anti-inflammatory effects of ivermectin in mouse model of allergic asthma. Inflamm Res. 2011;60(6): 589-96.

19. Rheinwald JG, Green H. Formation of a keratinizing epithelium in culture by a cloned cell line derived from a teratoma. Cell. 1975;6(3):317-30.

20. Rheinwald JG, Green H. Serial cultivation of strains of human epidermal keratinocytes: the formation of keratinizing colonies from single cells. Cell. 1975;6(3):331-43.

21. Prunieras M, Regnier M, Schlotterer M. New procedure for culturing human epidermal cells on allogenic or xenogenic skin: preparation of recombined grafts. Ann Chir Plast. 1979;24(4):357-62.

22. Morizane S, Yamasaki K, Kabigting FD, Gallo RL. Kallikrein expression and cathelicidin processing are independently controlled in keratinocytes by calcium, vitamin $\mathrm{D}(3)$, and retinoic acid. J Invest Dermatol. 2010;130(5):1297-306.

23. Zhang X, Song Y, Ci X, An N, Ju Y, Li H, et al. Ivermectin inhibits LPS-induced production of inflammatory cytokines and improves LPS-induced survival in mice. Inflamm Res. 2008;57(11):524-9.

24. Hoffmann E, Dittrich-Breiholz O, Holtmann $\mathrm{H}$, Kracht M. Multiple control of interleukin-8 gene expression. J Leukoc Biol. 2002;72(5):847-55.

25. Mahalingam S, Karupiah G. Chemokines and chemokine receptors in infectious diseases. Immunol Cell Biol. 1999;77(6):469-75.

26. Niyonsaba F, Ushio H, Nakano N, Ng W, Sayama K, Hashimoto K, et al. Antimicrobial peptides human beta-defensins stimulate epidermal keratinocyte migration, proliferation and production of proinflammatory cytokines and chemokines. J Invest Dermatol. 2007;127(3):594-604.

27. Stein Gold L, Kircik L, Fowler J, Jackson JM, Tan J, Draelos Z, et al. Long-term safety of ivermectin 1\% cream vs azelaic acid 15\% gel in treating inflammatory lesions of rosacea: results of two 40-week controlled, investigator-blinded trials. J Drugs Dermatol. 2014;13(11):1380-6. 
28. Taieb A, Khemis A, Ruzicka T, Baranska-Rybak W, Berth-Jones J, Schauber J, et al. Maintenance of remission following successful treatment of papulopustular rosacea with ivermectin 1\% cream vs. metronidazole $0.75 \%$ cream: 36-week extension of the ATTRACT randomized study. J Eur Acad Dermatol Venereol. 2016;30(5):829-36. 